\title{
Description of a new actinosporean type from South African freshwaters
}

\author{
Csaba Székely ${ }^{1, *}$, Annemarie Avenant-Oldewage ${ }^{2}$, Kálmán Molnár $^{1}$
}

\author{
${ }^{1}$ Veterinary Medical Research Institute, Hungarian Academy of Sciences, PO Box 18, 1518 Budapest, Hungary \\ ${ }^{2}$ Rand Afrikaans University, Department of Zoology, PO Box 524 Auckland Park, 2006 Johannesburg, South Africa
}

\begin{abstract}
Actinospore infection of oligochaetes collected from the mud of 2 freshwater biotopes in South Africa was studied. Using the 'cell-well plate method', a new aurantiactinomyxon type was found in $1.1 \%$ of the examined Branchiura sowerbyi oligochaete specimens from the Rietvlei River, north of Johannesburg, Gauteng, South Africa. In $1.5 \%$ of B. sowerbyi collected in a pond (Padda Dam), near the Rand Afrikaans University, Johannesburg, the same aurantiactinomyxon type was found. Infected oligochaetes were found only after collection and no actinosporean release was recorded in Branchiura specimens kept alive for several weeks. Actinospore infection showed a high intensity in oligochaetes in both positive cases. Until now, no actinosporean stages of myxosporeans have been described from South Africa. The aurantiactinospore type presented in this communication differs from the known types already described in the literature.
\end{abstract}

KEY WORDS: Myxosporea · Aurantiactinomyxon · Actinospore · Branchiura sowerbyi - Oligochaete · Alternate host · Padda Dam · Gauteng · Rietvlei River · South Africa

Resale or republication not permitted without written consent of the publisher

\section{INTRODUCTION}

The first report on actinospores described these organisms as parasites related to myxosporeans (Štolc 1899). For a long time, this group of parasites was believed to represent an independent taxonomic entity. Research on actinosporeans became more intensive after Wolf \& Markiw (1984) proved that they corresponded to the intraoligochaete developmental stages of fish-parasitic myxosporeans (Kent et al. 2001). The relevant research includes earlier surveys (Janiszewska 1957, Ormieres \& Frezil 1969, Marques 1984) as well as studies of actinospore infection of oligochaetes in natural waters and fish farms in connection with the life cycle of myxosporeans (Kent et al. 2001). Relatively few data are available on myxosporean infections of African freshwater fish (Fantham 1930, Fomena \& Bouix 1994, Reed 2002) and only a single paper appeared in Africa on their actinosporean alternative stages infecting oligochaetes (El-Mansy 2001).

Here, a new aurantiactinosporean stage of myxosporeans is reported from an oligochaete host from 2 freshwater biotopes in South Africa.

\section{MATERIALS AND METHODS}

Survey. The survey was conducted in 2 biotopes: (1) At 3 sampling points (Sites 1, 2 and 3) of the Rietvlei River, north of Johannesburg, where mainly Clarias gariepinus (Burchell, 1822) and Barbus polylepis Boulenger, 1970 are found. Other abundant fish species living in the river are Cyprinus carpio Linné, Tilapia sparrmanii Smith, 1840, Pseudocrenilabrus philander (Weber, 1897). (2) In a pond (Padda Dam), near the Rand Afrikaans University, Johannesburg, where the most abundant fish were Aplocheilichthys johnstoni Günther, 1893, P. philander (Weber, 1897), T. sparrmanii Smith, 1840, and Clarias gariepinus (Burchell, 1822), and the introduced C. carpio Linné is also abundant. All sampling was conducted in the second half of September 2003 before the rainy summer season at a water temperature of $20^{\circ} \mathrm{C}$. To detect the intraoligochaete actinospore types, mud samples were collected and transported to the fish parasitology laboratory of the Rand Afrikaans University. The oligochaetes were washed out from the mud with a net and gently picked up by forceps. After collection, the oligochaetes were individually placed into wells of 
cell-well plates (according to Yokoyama et al. 1991). Starting the following day, the water layer above each oligochaete in each of the wells was examined daily for $3 \mathrm{~d}$ for the presence of released actinospores. Then, the oligochaetes were kept in plastic tubes for $1 \mathrm{wk}$ and the water in each tube was changed daily in order to keep the oligochaetes alive. The oligochaetes were then transported to Hungary and monitored for actinospore release for an additional 2 mo.

If examination with a stereomicroscope indicated the presence of spores, the actinospores floating or adhering to the walls of the cell-well plates were examined on a slide with a light microscope at higher magnification. Microphotographs were then taken of the spores using a Zeiss Axioplan 2 compound microscope attached to a computer and the digital pictures were archived by Axiovision software. Subsequently, drawings were made of the actinospores and their measurements were taken. The characteristic dimensions of actinospores (size of polar capsules, diameter of spore body, length and width of caudal processes, largest span of processes) and the number of secondary cells in the sporoplasm were recorded by measuring newly released spores according to the guidelines suggested by Lom et al. (1997). To determine the dimensions of actinospores, measurements of 10 mature spores were averaged. A part of the released actinospores were fixed in $80 \%$ ethanol and transported to Hungary for further morphological studies. Measurements of spores were calculated from specimens on digitised photographs in South Africa and some additional measurements were made on 20 actinospore specimens fixed in ethanol. The measurements of these latter spores were measured with the help of a WinImago ${ }^{\circledR}$ program. Some of the representative oligochaetes from all the examined biotopes were fixed in $80 \%$ ethanol and identified in Estonia as described by Timm (1997).

For histological sections, infected oligochaetes were fixed in $10 \%$ neutral buffered formalin and embedded in paraplast-wax. Five $\mu \mathrm{m}$ thick sections were made and stained with haematoxylin and eosin. Photomicrographs of the sections were taken with an Olympus DH-10 digital camera mounted on an Olympus BH2 microscope.

\section{RESULTS}

\section{Survey}

Samples sent for identification and representing all of the 4 collection sites proved to contain developed and juvenile forms of Branchiura sowerbyi. Of the 93 examined oligochaetes from the 3 stretches of the Rietvlei River, only 1 large-sized B. sowerbyi specimen
$(1.1 \%)$ collected from Site 3 proved to be infected with a new aurantiactinomyxon type (Fig. 1). Another actinosporean release was found in a single specimen of the 65 B. sowerbyi oligochaetes (1.5\%) collected from Padda Dam (Fig. 2). In both cases, aurantiactinomyxon types were released. All the spores were released during the first day, after collection of the oligochaetes from the mud, into the wells of the cell-well plate. No further actinospore release was found in other oligochaete specimens from the same biotope during the 2 mo observation period.

\section{Effect of ethanol fixation on the secondary cells of the sporoplasm}

Secondary cells in sporoplasms of actinospores were not distinguishable in living aurantiactinospores (Fig. 3). However, in spores fixed in $80 \%$ ethanol and examined 1 mo later, contours of the secondary cells became well defined and the number of secondary cells as well as the polar capsules could be exactly counted (Fig. 4).

\section{Histology}

Early developmental stages and pansporocysts were located in the intestinal epithelium of the oligochaete, deforming and enlarging the normally monolayered epithelium (Fig. 5). At the infected part of the gut, almost each epithelial cell contained pansporocysts of different developmental stages. In some of the pansporocysts, the 8 developing actinospores were clearly visible. In the more developed pansporocysts, however, 4 actinospores were cross-sectioned at the level of the sporoplasm and revealed the presence of secondary cells. Uninfected epithelial cells were encountered only in small stretches around the intestinal lumen of the worms. In other parts of the degenerated epithelium, nuclei of the deformed epithelial cells were jammed between the spaces of pansporocysts (Fig. 6).

\section{Description of the detected new actinospore type}

From both infected Branchiura specimens of the 2 sampling sites, similar aurantiactinospores were released. Only minor non-significant differences were observed, both in terms of the dimensions and form of the spores. This aurantiactinomyxon type, which differs from the already known actinospores, has been described as follows on the basis of spores collected from the Branchiura specimen from Padda Dam 

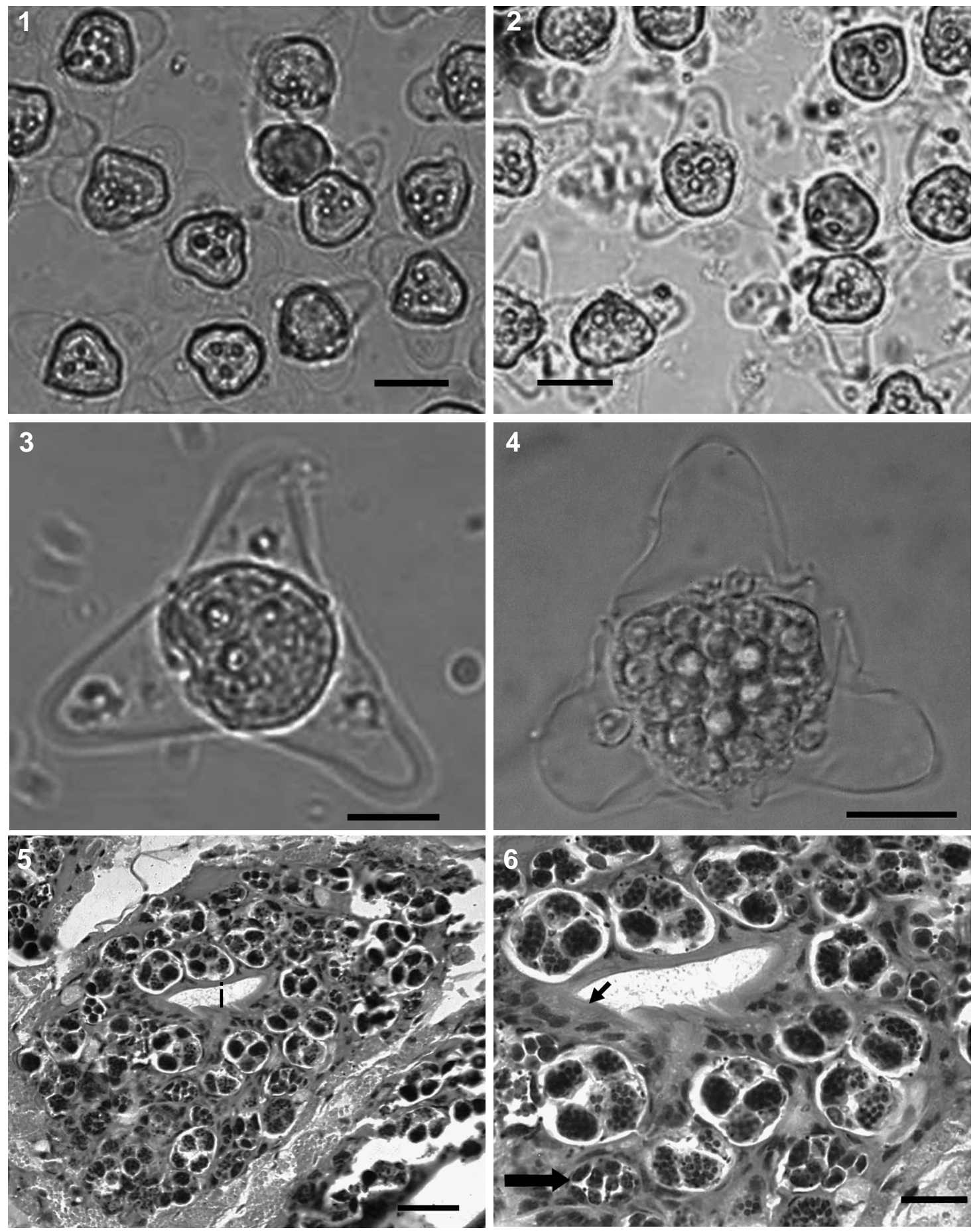

Fig. 1. Aurantiactinospores released from a Branchiura sowerbyi specimen collected from the Rietvlei River. Scale bar $=20 \mu \mathrm{m}$.

Fig. 2. Aurantiactinospores released from a B. sowerbyi specimen collected at Padda Dam. Scale bar $=20 \mu \mathrm{m}$. Fig. 3 . Enlarged

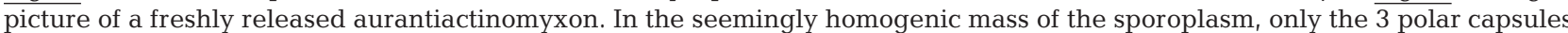
are seen. The nuclei of the caudal projections can also be seen. Scale bar $=10 \mu \mathrm{m}$. Fig. 4 . Aurantiactinospores fixed in $80 \%$ ethanol. Besides nuclei of the caudal projections, secondary cells of the sporoplasm are also seen. Scale bar $=10 \mu \mathrm{m}$. Fig. 5 . Histological section of a $B$. sowerbyi oligochaete. In each segment, jammed cells of the proliferated epithelium containing aurantiactinomyxon developmental stages are located around the intestinal lumen (i). H \& E. Scale bar $=15 \mu \mathrm{m}$. Fig. 6 . Enlarged part of Fig. 5. Remnants of the relatively unaffected epithelial cells are found only in a short stretch around the intestinal lumen (small arrow). Most of the epithelial cells are infected with pansporocysts of an aurantiactinomyxon type. In younger pansporocysts (large arrow), 8 developing spores can be detected. In more developed pansporocysts, only 4 spores locating in 1 level and containing secondary cells are seen. H \& E. Scale bar $=30 \mu \mathrm{m}$ 


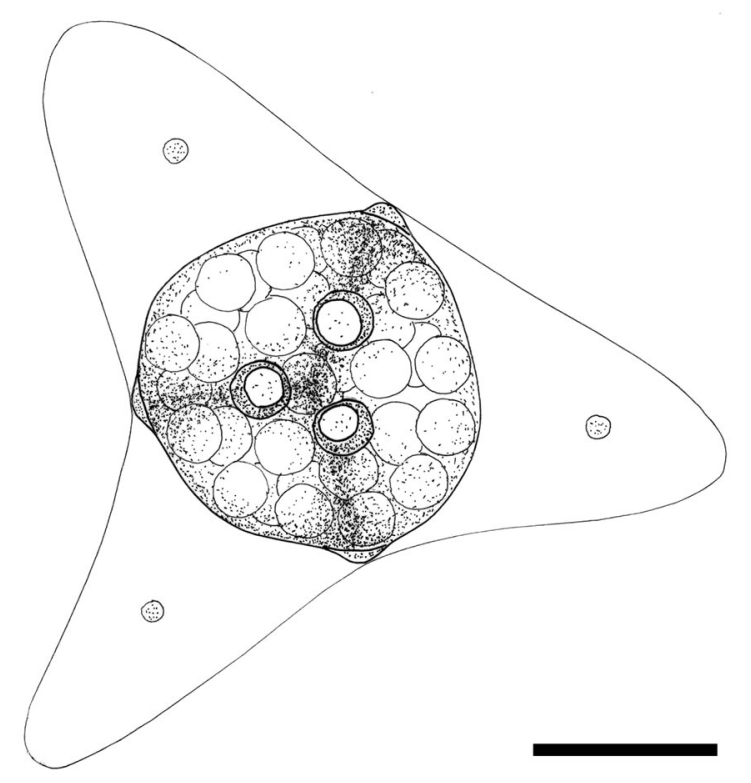

Fig. 7. Schematic drawing of the aurantiactinospore type released by a Branchiura sowerbyi specimen from Padda Dam. Scale bar $=10 \mu \mathrm{m}$

(Fig. 7). Minor variations observed in morphology and size between 2 oligochaetes are presented in Table 1.

Aurantiactinomyxon type

(Figs. 2 \& 7, Table 1: Type 2).

Spores always single, epispore without style with 3 leaf-like short caudal processes of equal length. Spores released from Branchiura sowerbyi. Spore body $19.6 \mu \mathrm{m}$ (18.1 to 21.8) in diameter with 3 apical polar capsules $2.7 \mu \mathrm{m}$ (2.6 to 2.9) in diameter. Secondary cells, 64. Three caudal processes $10.5 \mu \mathrm{m}$ (9.9 to 17.4) long and $15.2 \mu \mathrm{m}$ (13.3 to 18.7 ) wide.

\section{DISCUSSION}

Aurantiactinomyxon types as such or as the alternative spore forms of myxosporeans have been described by the authors listed in Table 1 . The aurantiactinomyxon type detected in this study differs substantially from the types already described in at least 1 of its dimensions.

Based on the measurements described in Table 1, the aurantiactinospores detected in this study, although collected from 2 different biotopes, appear to be identical and represent a new form hitherto not described.

The aurantiactinomyxon type found during this survey differs from the over 40 known types not only in its measurements and shape, but in the majority of cases also by the oligochaete alternate hosts. Until now, only
Yokoyama et al. (1993), Yokoyama (1997), El-Mansy et al. (1998a,b) and Székely et al. (2000) have recorded aurantiactinomyxon types of the relatively large-sized Branchiura sowerbyi. The prevalence of actinosporean infection was relatively low, significantly less than found by El-Mansy et al. (1998b) in Lake Balaton or by Székely et al. (2003) in Japan, but corresponded to results obtained in other similar surveys (Xiao \& Desser 1998, Negredo \& Mulcachy 2001, Özer et al. 2002 and Oumona et al. 2003). The low prevalence only represents a temporary situation as Gilbert \& Granath (2001) proved that an oligochaete can repeatedly release actinospores after a long dormitory period. Furthermore, our unpublished observations prove that Branchiura specimens brought to the laboratory from natural waters preserve their infectiveness and that even without re-infection, they are able to release actinosporeans 18 to 24 mo after being collected. It is not excluded, therefore, that Branchiura specimens brought from South Africa and kept alive in Hungary might produce auroactinospores or other actinospores in the future.

Experts in the systematics of myxosporeans disagree concerning the description of new actinospore types detected from oligochaetes. According to Kent \& Lom (1999), actinospores detected from oligochaetes should be regarded as developmental stages of Myxozoa species parasitising vertebrate hosts, primarily fish, whereas Lester et al. (1998) are of the opinion that new species of Myxozoa can be described based solely on the actinospores. Considering their arguments, we accept the guidelines provided by Kent \& Lom (1999) and describe new actinospores only as types in every case. Their description as species should be based on the production of myxospores, experimentally obtained in the vertebrate host or on their identification by molecular biological methods.

When describing actinosporean types, one has to be especially careful. Morphological characterization of the forms found is extremely important but in itself not enough. One cannot know whether the shape of the actinospores is as stable as in the case of myxospores. Hallett et al. (2002) stated that the molecular patterns of 2 morphologically clearly differing actinosporean types proved to be genetically identical. On the other hand, we cannot be sure that 2 morphologically similar actinosporean types, with identical dimensions, would be identical at the molecular biological level.

In the identification and description of actinosporean stages, the number of secondary cells in the sporoplasm is a very important key. These numbers are usually multiples of $2(4,8,16,32$, etc.). Unfortunately, counting the numbers of these cells is not easy; therefore, for most of the described actinosporean types, this detail was not provided or only an approximate number was given. In fresh spores, the structure of the 
Table 1. Comparison of measurements of aurantiactinomyxon type spores detected during the present study and those found in the scientific literature. nd: not determined; l.: length; w.: width $(\mu \mathrm{m})$

\begin{tabular}{|c|c|c|c|c|c|c|c|c|}
\hline Source & Locality & $\begin{array}{l}\text { Aurantiactinomyxon } \\
\text { type/species or } \\
\text { myxosporean } \\
\text { form/fish host }\end{array}$ & $\begin{array}{l}\text { Length of } \\
\text { caudal process } \\
\text { (range); width of } \\
\text { caudal process at } \\
\text { the basis (range) }\end{array}$ & $\begin{array}{l}\text { Polar capsule } \\
\text { l. (range); } \\
\text { w. (range) }\end{array}$ & $\begin{array}{l}\text { Diameter } \\
\text { of spore } \\
\text { body } \\
\text { (range) }\end{array}$ & $\begin{array}{l}\text { Largest } \\
\text { span } \\
\text { (range) }\end{array}$ & $\begin{array}{l}\text { No. } \\
\text { secondary } \\
\text { cells }\end{array}$ & $\begin{array}{l}\text { Alternate } \\
\text { host }\end{array}$ \\
\hline Present study & $\begin{array}{l}\text { South Africa, } \\
\text { Rietvlei River }\end{array}$ & Type 1 & $\begin{array}{c}10.4(9.8-16.9) \\
15.0(13.1-18.2)\end{array}$ & $2.7(2.6-2.8)$ & $19.5(17.9-21.4)$ & $\begin{array}{c}36.7 \\
(32-39.2)\end{array}$ & nd & $\begin{array}{l}\text { Branchiura } \\
\text { sowerbyi }\end{array}$ \\
\hline Present study & $\begin{array}{l}\text { South Africa, } \\
\text { Padda Dam } \\
\text { Johannesburg }\end{array}$ & Type 2 & $\begin{array}{c}10.5(9.9-17.4) \\
15.2(13.3-18.7)\end{array}$ & $2.7(2.6-2.9)$ & $19.6(18.1-21.8)$ & $\begin{array}{c}35.8 \\
(30.2-41.3)\end{array}$ & 64 & B. sowerbyi \\
\hline $\begin{array}{l}\text { Janiszewska } \\
(1957)\end{array}$ & $\begin{array}{c}\text { Poland, } \\
\text { River Ropa }\end{array}$ & $\begin{array}{l}\text { A. raabei } \\
\text { iunioris }\end{array}$ & $\begin{array}{l}25-30 \\
\text { nd }\end{array}$ & nd & 17 & nd & 16 & $\begin{array}{l}\text { Limnodrilus } \\
\text { hoffmeisteri }\end{array}$ \\
\hline $\begin{array}{l}\text { Ormières } \\
(1968)\end{array}$ & France? & A. pavinsis & $\begin{array}{c}12(10-14) \\
\text { nd }\end{array}$ & $\begin{array}{l}3(2.5-3.5) \\
2(1.5-2.5)\end{array}$ & $10(9-11)$ & nd & 12 & $\begin{array}{l}\text { Stylodrylus } \\
\text { heringianus }\end{array}$ \\
\hline $\begin{array}{l}\text { Ormières \& Frézil } \\
\text { (1969) }\end{array}$ & $\begin{array}{l}\text { Yugoslavia, } \\
\text { Lake Ochrid }\end{array}$ & A. eiseniellae & $\begin{array}{c}10-15 \\
\text { nd }\end{array}$ & nd & 12 & nd & 32 & $\begin{array}{l}\text { Eiseniella } \\
\text { tetraedra }\end{array}$ \\
\hline Marques (1984) & France & A. pavinsis & $10-12(15-20)$ & nd & $8(12)$ & nd & 16 & $\begin{array}{l}\text { Stylodrylus } \\
\text { heringianus }\end{array}$ \\
\hline Marques (1984) & France & A. stellans & $70-90$ & nd & $15-20$ & nd & 16 & Tubifex spp. \\
\hline Marques (1984) & France & A. trifolium & $40-50$ & nd & $20-25$ & nd & 32 & Tubifex spp. \\
\hline Burtle et al. (1991) & $\begin{array}{l}\text { USA, } \\
\text { catfish pond }\end{array}$ & $\begin{array}{l}\text { Aurantiactinomyxon/ } \\
\text { PGD organism/ } \\
\text { channel catfish }\end{array}$ & $\begin{array}{c}29.5 \\
\text { nd }\end{array}$ & nd & 19.5 & nd & $>32$ & Dero digitata \\
\hline $\begin{array}{l}\text { Bartholomew } \\
\text { et al. (1992) }\end{array}$ & $\begin{array}{l}\text { USA, } \\
\text { experimental } \\
\text { tanks }\end{array}$ & Type 1 & nd & nd & nd & nd & nd & $\begin{array}{l}\text { Nais } \\
\text { bretscheri }\end{array}$ \\
\hline $\begin{array}{l}\text { El-Matbouli } \\
\text { et al. (1992) }\end{array}$ & $\begin{array}{l}\text { Germany, } \\
\text { experimental } \\
\text { infection }\end{array}$ & $\begin{array}{c}\text { Hofferellus } \\
\text { carassii } \\
\text { Goldfish }\end{array}$ & $\begin{array}{c}(25) \\
\text { nd }\end{array}$ & nd & (13) & nd & nd & $\begin{array}{l}\text { Mixed species: } \\
\text { T. tubifex, } \\
\text { T. ignotus } \\
\text { and L. } \\
\text { hoffmeisteri }\end{array}$ \\
\hline $\begin{array}{l}\text { Grossheider \& } \\
\text { Körting (1992) }\end{array}$ & $\begin{array}{l}\text { Germany, } \\
\text { experimental } \\
\text { infection }\end{array}$ & $\begin{array}{l}\text { H. cyprini } \\
\text { Common carp }\end{array}$ & $\begin{array}{c}(30) \\
\text { nd }\end{array}$ & nd & $(15)$ & nd & nd & Nais spp. \\
\hline Styer et al. (1991) & $\begin{array}{l}\text { USA, } \\
\text { experimental } \\
\text { infection }\end{array}$ & $\begin{array}{c}\text { A. major } \\
\text { PGD/ } \\
\text { channel catfish }\end{array}$ & $11 \times 36$ & nd & $18-22$ & nd & nd & D. digitata \\
\hline Styer et al. (1991) & $\begin{array}{l}\text { USA, } \\
\text { experimental } \\
\text { infection }\end{array}$ & $\begin{array}{c}\text { A. minor } \\
\text { PGD/ } \\
\text { channel catfish }\end{array}$ & 36 & nd & $13-16$ & nd & nd & D. digitata \\
\hline $\begin{array}{l}\text { Benajiba \& } \\
\text { Marques (1993) }\end{array}$ & $\begin{array}{c}\text { France, } \\
\text { experimental } \\
\text { infection in tanks }\end{array}$ & $\begin{array}{l}\text { Myxidium giardi } \\
\text { European } \\
\text { eel }\end{array}$ & $\begin{array}{c}(20) \\
\text { nd }\end{array}$ & nd & (12) & nd & nd & Tubifex spp. \\
\hline $\begin{array}{l}\text { Pote \& } \\
\text { Waterstrat (1993) }\end{array}$ & $\begin{array}{l}\text { USA (Mississippi } \\
\text { State), } \\
\text { Fish Pond }\end{array}$ & $\begin{array}{l}\text { PGD organism/ } \\
\text { channel catfish } \\
\text { Ictalurus punctatus }\end{array}$ & $\begin{array}{l}26(21-32) \\
11(8-12)\end{array}$ & nd & $23(20-24)$ & nd & nd & D. digitata \\
\hline $\begin{array}{l}\text { Trouillier et al. } \\
\text { (1996) }\end{array}$ & $\begin{array}{l}\text { Germany, } \\
\text { experimental } \\
\text { tank }\end{array}$ & $\begin{array}{c}\text { Aurantiactinomyxon/ } \\
\text { H. carassii/ } \\
\text { goldfish }\end{array}$ & $\begin{array}{c}48.8(40.6-57) \\
11.7(10.2-13.3)\end{array}$ & nd & $23.5(20.4-26.6)$ & nd & nd & Nais elinguis \\
\hline Yokoyama (1993) & $\begin{array}{c}\text { Japan, } \\
\text { goldfish pond }\end{array}$ & $\begin{array}{l}\text { Aurantiactinomyxon } \\
\text { spp. } 1\end{array}$ & $\begin{array}{l}16 \\
\text { nd }\end{array}$ & nd & 11 & nd & 8 & B. sowerbyi \\
\hline $\begin{array}{l}\text { McGeorge et al. } \\
(1997)\end{array}$ & $\begin{array}{c}\text { Scotland, } \\
\text { Salmon hatchery } \\
\text { stream }\end{array}$ & $\begin{array}{c}\text { Aurantiactinomyxon/ } \\
\text { Sphaerospora } \\
\text { truttae }\end{array}$ & $\begin{array}{c}25.6(19-31) \\
\text { nd }\end{array}$ & $2.7(2-3)$ & $13.7(12-15)$ & nd & nd & $\begin{array}{l}\text { Immature } \\
\text { tubificid }\end{array}$ \\
\hline Yokoyama (1997) & Japan & $\begin{array}{c}\text { Thelohanellus } \\
\text { hovorkai } \\
\text { Common carp (koi) }\end{array}$ & $\begin{array}{c}25-33 \\
\text { nd }\end{array}$ & nd & $18-22$ & nd & 32 & B. sowerbyi \\
\hline $\begin{array}{l}\text { El-Mansy } \\
\text { et al. (1998a) }\end{array}$ & $\begin{array}{l}\text { Hungary, } \\
\text { fish pond }\end{array}$ & NO 1 & 17.5 & $2 \times 2$ & 18.3 & 45.4 & nd & T. tubifex \\
\hline $\begin{array}{l}\text { El-Mansy } \\
\text { et al. (1998a) }\end{array}$ & $\begin{array}{l}\text { Hungary, } \\
\text { fish pond }\end{array}$ & NO 2 & $\begin{array}{l}65.7 \\
10.5\end{array}$ & $4 \times 1.7$ & 22.8 & 142.5 & nd & $\begin{array}{l}\text { Water, } \\
\text { B. sowerbyi }\end{array}$ \\
\hline $\begin{array}{l}\text { El-Mansy } \\
\text { et al. (1998a) }\end{array}$ & $\begin{array}{l}\text { Hungary, } \\
\text { fish pond }\end{array}$ & NO 3 & $\begin{array}{c}70.3 \\
8\end{array}$ & $2.9 \times 2.9$ & 22.8 & 149.3 & nd & $\begin{array}{l}\text { Water, } \\
\text { B. sowerbyi }\end{array}$ \\
\hline $\begin{array}{l}\text { El-Mansy } \\
\text { et al. (1998a) }\end{array}$ & $\begin{array}{l}\text { Hungary, } \\
\text { fish pond }\end{array}$ & NO 4 & $\begin{array}{l}55.7 \\
11.2\end{array}$ & $2.9 \times 2.9$ & 19.4 & 122 & nd & $\begin{array}{l}\text { Water, } \\
\text { B. sowerbyi }\end{array}$ \\
\hline
\end{tabular}


Table 1 (continued)

\begin{tabular}{|c|c|c|c|c|c|c|c|c|}
\hline Source & Locality & $\begin{array}{l}\text { Aurantiactinomyxon } \\
\text { type/species or } \\
\text { myxosporean } \\
\text { form/fish host }\end{array}$ & $\begin{array}{l}\text { Length of } \\
\text { caudal process } \\
\text { (range); width of } \\
\text { caudal process at } \\
\text { the basis (range) }\end{array}$ & $\begin{array}{l}\text { Polar capsule } \\
\text { l. (range); } \\
\text { w. (range) }\end{array}$ & $\begin{array}{l}\text { Diameter } \\
\text { of spore } \\
\text { body } \\
\text { (range) }\end{array}$ & $\begin{array}{l}\text { Largest } \\
\text { span } \\
\text { (range) }\end{array}$ & $\begin{array}{l}\text { No. } \\
\text { secondary } \\
\text { cells }\end{array}$ & $\begin{array}{l}\text { Alternate } \\
\text { host }\end{array}$ \\
\hline $\begin{array}{l}\text { El-Mansy } \\
\text { et al. (1998a) }\end{array}$ & $\begin{array}{l}\text { Hungary, } \\
\text { fish pond }\end{array}$ & NO 5 & $\begin{array}{c}17.2 \\
3.9\end{array}$ & $1.4 \times 1.4$ & 9.9 & 39.5 & nd & $\begin{array}{l}\text { Water, } \\
\text { B. sowerbyi }\end{array}$ \\
\hline $\begin{array}{l}\text { El-Mansy } \\
\text { et al. (1998a) }\end{array}$ & $\begin{array}{l}\text { Hungary, } \\
\text { fish pond }\end{array}$ & NO 6 & $\begin{array}{l}24.2 \\
11.2\end{array}$ & $2.8 \times 2.8$ & 19.7 & 55.6 & nd & $\begin{array}{l}\text { Limnodrilus } \\
\text { spp. }\end{array}$ \\
\hline $\begin{array}{l}\text { El-Mansy } \\
\text { et al. (1998a) }\end{array}$ & $\begin{array}{l}\text { Hungary, } \\
\text { fish pond }\end{array}$ & NO 7 & $\begin{array}{c}24.4 \\
9.5\end{array}$ & $2.8 \times 2.5$ & 18.9 & 58.4 & nd & Water \\
\hline $\begin{array}{l}\text { El-Mansy } \\
\text { et al. (1998a) }\end{array}$ & $\begin{array}{l}\text { Hungary, } \\
\text { fish pond }\end{array}$ & NO 8 & $\begin{array}{c}12.2 \\
9\end{array}$ & $1.4 \times 1.4$ & 22.6 & 39.8 & nd & $\begin{array}{c}\text { Limnodrilus } \\
\text { spp. }\end{array}$ \\
\hline $\begin{array}{l}\text { El-Mansy } \\
\text { et al. (1998a) }\end{array}$ & $\begin{array}{l}\text { Hungary, } \\
\text { fish pond }\end{array}$ & NO 9 & $\begin{array}{c}51.3 \\
9.5\end{array}$ & $2.3 \times 2.3$ & 18.8 & 103.2 & nd & $\begin{array}{l}\text { Water, } \\
\text { B. sowerbyi }\end{array}$ \\
\hline $\begin{array}{l}\text { El-Mansy } \\
\text { et al. (1998a) }\end{array}$ & $\begin{array}{l}\text { Hungary, } \\
\text { fish pond }\end{array}$ & NO 10 & $\begin{array}{c}16.7 \\
8.8\end{array}$ & $1.7 \times 1.7$ & 15.5 & 39.5 & nd & $\begin{array}{l}\text { Water, } \\
\text { B. sowerbyi }\end{array}$ \\
\hline $\begin{array}{l}\text { El-Mansy } \\
\text { et al. (1998a) }\end{array}$ & $\begin{array}{l}\text { Hungary, } \\
\text { fish pond }\end{array}$ & NO 11 & $\begin{array}{c}31.9 \\
3.7\end{array}$ & $3.4 \times 2$ & 8.5 & 46.5 & nd & Water \\
\hline $\begin{array}{l}\text { El-Mansy } \\
\text { et al. (1998a) }\end{array}$ & $\begin{array}{l}\text { Hungary, } \\
\text { fish pond }\end{array}$ & NO 12 & $\begin{array}{c}26.5 \\
8.7\end{array}$ & $2.8 \times 3.1$ & 12.1 & 59.2 & nd & $\begin{array}{l}\text { Water, } \\
\text { B. sowerbyi }\end{array}$ \\
\hline $\begin{array}{l}\text { El-Mansy } \\
\text { et al. (1998b) }\end{array}$ & $\begin{array}{l}\text { Hungary, } \\
\text { Lake Balaton }\end{array}$ & NO 1 & $\begin{array}{c}51.3 \\
9.5\end{array}$ & $2.3 \times 2.3$ & 18.8 & 103.2 & nd & $\begin{array}{l}\text { Water, } \\
\text { B. sowerbyi }\end{array}$ \\
\hline $\begin{array}{l}\text { El-Mansy } \\
\text { et al. (1998b) }\end{array}$ & $\begin{array}{l}\text { Hungary, } \\
\text { Lake Balaton }\end{array}$ & NO 2 & $\begin{array}{l}22.5 \\
11.7\end{array}$ & $2.8 \times 2$ & 21.1 & 52.2 & nd & Limnodrilus \\
\hline $\begin{array}{l}\text { El-Mansy } \\
\text { et al. (1998b) }\end{array}$ & $\begin{array}{l}\text { Hungary, } \\
\text { Lake Balaton }\end{array}$ & NO 3 & $\begin{array}{c}17.2 \\
3.9\end{array}$ & $1.4 \times 1.4$ & 9.9 & 39.5 & nd & $\begin{array}{l}\text { Water, } \\
\text { B. sowerbyi }\end{array}$ \\
\hline $\begin{array}{l}\text { Székely } \\
\text { et al. (1998) }\end{array}$ & $\begin{array}{l}\text { Hungary, } \\
\text { experimental } \\
\text { infection }\end{array}$ & $\begin{array}{l}\text { T. nikolskii/ } \\
\text { common carp }\end{array}$ & $\begin{array}{l}13.4(11.3-15.5) \\
\quad 9(8.5-9.6)\end{array}$ & $\begin{array}{l}2.1(2-2.2) \\
2.1(2-2.2)\end{array}$ & $\begin{array}{c}21.1 \\
(21-21.2)\end{array}$ & $\begin{array}{c}40.5 \\
(40-41)\end{array}$ & 16 & T. tubifex \\
\hline $\begin{array}{l}\text { Székely } \\
\text { et al. (1998) }\end{array}$ & $\begin{array}{l}\text { Hungary, } \\
\text { experimental } \\
\text { infection }\end{array}$ & $\begin{array}{l}\text { T. hovorkai/ } \\
\text { Common carp }\end{array}$ & $\begin{array}{l}29(28.2-29.6) ; \\
9.2(8.1-10.2)\end{array}$ & $\begin{array}{c}3.42 \\
(3.4-3.5) ; \\
3.36(3.3-3.4)\end{array}$ & $\begin{array}{c}18.6 \\
(18.3-18.9)\end{array}$ & $\begin{array}{c}65.2 \\
(65.1-65.3)\end{array}$ & 32 & B. sowerbyi \\
\hline $\begin{array}{l}\text { Xiao \& Desser } \\
\text { (1998) }\end{array}$ & $\begin{array}{c}\text { Canada, } \\
\text { Lake Sasajewun }\end{array}$ & Type 1 & $\begin{array}{c}24(21-26) ; \\
13-16\end{array}$ & $\begin{array}{c}3(2.7-3.4) \\
1.5(1.4-1.7)\end{array}$ & $\begin{array}{c}12(11.5-13.8)_{i} \\
11(10-12.5)\end{array}$ & nd & $64-128$ & L. hoffmeisteri \\
\hline $\begin{array}{l}\text { Lin et al. } \\
\text { (1999) }\end{array}$ & $\begin{array}{l}\text { USA, } \\
\text { catfish pond }\end{array}$ & $\begin{array}{l}\text { Aurantiactinomyxon } \\
\text { janisewskai/Henneguya } \\
\text { exilis/channel catfish }\end{array}$ & nd & nd & nd & nd & nd & D. digitata \\
\hline $\begin{array}{l}\text { Székely } \\
\text { et al. (2000) }\end{array}$ & $\begin{array}{c}\text { Spain, } \\
\text { Mijares River/ } \\
\text { Sitjar Reservoir }\end{array}$ & Type 1 & $\begin{array}{l}6.1(4.8-7.3) ; \\
5.6(4.8-6.5)\end{array}$ & $\begin{array}{c}1.6(1.5-1.7)_{i} \\
1.1(1-1.1)\end{array}$ & $8.1(7.3-8.9)$ & $\begin{array}{c}17.1 \\
(16.1-18.5)\end{array}$ & 64 & B. sowerbyi \\
\hline $\begin{array}{l}\text { Negredo \& } \\
\text { Mulcachy (2001) }\end{array}$ & $\begin{array}{l}\text { Ireland, } \\
\text { Cloonee River }\end{array}$ & $\begin{array}{l}\text { Aurantiactino- } \\
\text { myxon } 1\end{array}$ & $\begin{array}{c}21.1(18.2-23.4) \\
16.1(13-19.5)\end{array}$ & $3(2.5-3.9)$ & $14.4(12.6-16.9)$ & nd & nd & T. ignotus \\
\hline $\begin{array}{l}\text { Negredo \& } \\
\text { Mulcachy (2001) }\end{array}$ & $\begin{array}{c}\text { Ireland, } \\
\text { Cloonee River }\end{array}$ & $\begin{array}{l}\text { Aurantiactino- } \\
\text { myxon } 2\end{array}$ & $\begin{array}{c}31(26-36) \\
10.6(9.1-13)\end{array}$ & $2.7(2.6-3.1)$ & $14.1(13-15.6)$ & nd & nd & L. hoffmeisteri \\
\hline $\begin{array}{l}\text { Negredo \& } \\
\text { Mulcachy (2001) }\end{array}$ & $\begin{array}{c}\text { Ireland, } \\
\text { Cloonee River }\end{array}$ & $\begin{array}{l}\text { Aurantiactino- } \\
\text { myxon } 3\end{array}$ & $\begin{array}{l}20.8(18.2-23.4) \\
10.4(10.4-10.4)\end{array}$ & nd & $9.1(9.1-9.1)$ & nd & nd & T. ignotus \\
\hline $\begin{array}{l}\text { Hallett et al. } \\
(2002)\end{array}$ & $\begin{array}{l}\text { Germany, } \\
\text { Bavaria }\end{array}$ & $\begin{array}{c}\text { Auratiactino- } \\
\text { myxon morphotype } 1\end{array}$ & $\begin{array}{l}37.3(28.5-49.2) \\
15.7(14.2-18.1)\end{array}$ & $3.1(2.6-3.9)$ & $19.4(16.8-21.4)$ & $\begin{array}{c}82.4 \\
(68.6-103.6)\end{array}$ & 30 & T. tubifex \\
\hline $\begin{array}{l}\text { Hallett et al. } \\
(2002)\end{array}$ & $\begin{array}{l}\text { Germany, } \\
\text { Bavaria }\end{array}$ & $\begin{array}{c}\text { Auratiactino- } \\
\text { myxon morphotype } 2\end{array}$ & $\begin{array}{c}87.7(75.1-103.6) \\
13.1(10.4-15.5)\end{array}$ & 3.1 & $19.7(18.1-22.0)$ & $\begin{array}{c}163.7 \\
(134.7-194.3)\end{array}$ & 30 & T. tubifex \\
\hline $\begin{array}{l}\text { Özer et al. } \\
(2002)\end{array}$ & $\begin{array}{l}\text { Scotland, } \\
\text { salmon farm }\end{array}$ & Type 1 & $32(31-36)$ & nd & $14.4(12-15)$ & nd & $64-128$ & T. tubifex \\
\hline $\begin{array}{l}\text { Özer et al. } \\
(2002)\end{array}$ & $\begin{array}{l}\text { Scotland, } \\
\text { salmon farm }\end{array}$ & Type 2 & $24.7(23.6-26.5)$ & nd & $14.9(14-18.7)$ & nd & 64 & T. tubifex \\
\hline $\begin{array}{l}\text { Özer et al. } \\
(2002)\end{array}$ & $\begin{array}{l}\text { Scotland, } \\
\text { salmon farm }\end{array}$ & Type 3 & 114.5 (101.4-124.8) & nd & $\begin{array}{c}24(23.4-24.9) \\
21.8(20.3-23.4)\end{array}$ & nd & 32 & T. tubifex \\
\hline $\begin{array}{l}\text { Özer et al. } \\
(2002)\end{array}$ & $\begin{array}{l}\text { Scotland, } \\
\text { salmon farm }\end{array}$ & Type 4 & $28.3(23.4-31.2)$ & nd & $11.9(11.2-14)$ & nd & 32 & T. tubifex \\
\hline $\begin{array}{l}\text { Oumuna et al. } \\
\text { (2003) }\end{array}$ & $\begin{array}{c}\text { Germany, } \\
\text { Bavarian fish farm }\end{array}$ & A. pavinsis & $\begin{array}{c}12(10-14) \\
\text { nd }\end{array}$ & $\begin{array}{l}3(2.5-3.5) \\
2(1.5-2.5)\end{array}$ & $10(9-11)$ & nd & 12 & $\begin{array}{l}\text { Water } \\
\text { T. tubifex? }\end{array}$ \\
\hline $\begin{array}{l}\text { Oumuna et al. } \\
\text { (2003) }\end{array}$ & $\begin{array}{c}\text { Germany, } \\
\text { Bavarian Fish Farm }\end{array}$ & $\begin{array}{l}\text { Aurantiactino- } \\
\text { myxon type } 1\end{array}$ & $76(75-77)$ & $\begin{array}{l}5(4.7-5.3) \\
4(3.8-4.2)\end{array}$ & $16(15-17)$ & nd & nd & Water \\
\hline $\begin{array}{l}\text { Székely } \\
\text { et al. (2003) }\end{array}$ & $\begin{array}{c}\text { Japan, } \\
\text { brook on Fuji Mountai } \\
\text { near Yamanashi }\end{array}$ & $\begin{array}{c}\text { Type 1 } \\
13.5(13-14)\end{array}$ & $\begin{array}{c}12.4(10-14) \\
1\end{array}$ & 2 & $13.5(13-14)$ & $26.8(25-30)$ & 8 & T. tubifex \\
\hline
\end{tabular}


sporoplasm is hardly seen, therefore the number of secondary cells was counted either by compressing the spore body (Székely et al. 1998, Molnár et al. 1999) or in histological sections (Alvarez-Pellitero et al. 2002). The observation that alcohol-preserved specimens reveal the contours of secondary cells well, might prove to be a new method for specialists working on actinosporeans.

Acknowledgements. The authors thank R. Greenfield, M. Matla, M. Ross and S. Tsabalala for their help in collecting oligochaetes as well as M. Barson for laboratory work. The study was financially supported by the Hungarian National Grants, OTKA T31755 and T42464 projects. Collection trips were funded by a grant from the National Research Foundation (SA) to A.A.O. We also thank P. O. Györgyi for pencildrawings and T. Timm for the identification of oligochaetes.

\section{LITERATURE CITED}

Alvarez-Pellitero P, Molnár K, Sitja-Bobadilla A, Székely C (2002) Comparative ultrastructure of the actinosporean stages of Myxobolus bramae and M. pseudodispar (Myxozoa). Parasitol Res 88:198-207

Bartholomew J, Rohovec JS, Fryer JL (1992) Ceratomyxa chasta infections of salmonid fish. In: Kimura T (ed) Proc OJI Int Symp Salmonid Diseases. Hokkaido University Press, Sapporo, p 267-275

Benajiba MH, Marques A (1993) The alternation of actinomyxidian and myxosporidian sporal forms in the development of Myxidium giardi (parasite of Anguilla anguilla) through oligochaetes. Bull Eur Assoc Fish Pathol 13: 100-103

Burtle GJ, Harrison LR, Styer EL (1991) Detection of a triactinomyxid myxozoan in an oligochaete from ponds with proliferative gill disease in channel catfish. J Aquat Anim Health 3:281-287

El-Mansy A (2001) First record of actinosporean stages of fish myxosporean parasites (Myxozoa: Myxosporea) in Egypt. J Egypt Soc Parasitol 31:449-465

El-Mansy A, Székely C, Molnár K (1998a) Studies on the occurrence of actinosporean stages of fish myxosporeans in a fish farm of Hungary, with the description of triactinomyxon, raabeia, aurantiactinomyxon and neoactinomyxon types. Acta Vet Hung 46:259-284

El-Mansy A, Székely C, Molnár K (1998b) Studies on the occurrence of actinosporean stages of myxosporeans in Lake Balaton, Hungary, with the description of triactinomyxon, raabeia and aurantiactinomyxon types. Acta Vet Hung 46:437-450

El-Matbouli M, Fischer-Scherl T, Hoffmann R (1992) Transmission of Hoferellus carassii Achmerov, 1960 to goldfish Carassius auratus via an aquatic oligochaete. Bull Eur Assoc Fish Pathol 12(2):54-56

Fantham HB (1930) Some parasitic protozoa found in South Africa. XIII. South Afr J Sci 27:376-390

Fomena A, Bouix G (1994) New Myxosporidia species (Myxozoa) from freshwater teleosts in southern Cameroon (Central Africa). J Afr Zool 108:481-491

Gilbert M, Granath W (2001) Persistent infection of Myxobolus cerebralis, the causative agent of salmonid whirling disease, in Tubifex tubifex. J Parasitol 87:101-107

Grossheider G, Körting W (1992) First evidence that Hoferellus cyprini (Doflein, 1898) is transmitted by Nais sp. Bull
Eur Ass Fish Pathol 12:17-20

Hallett SL, Atkinson SD, El-Matbouli M (2002) Molecular characterisation of two aurantiactinomyxon (Myxozoa) phenotypes reveals one genotype. J Fish Dis 25:627-631

Janiszewska J (1957) Actinomyxidia. II. New systematics, sexual cycle, description of new genera and species. Zool Pol 8:3-34

Kent M, Lom J (1999) Can a new species of Myxozoa be described based solely on their actinosporean stage? Parasitol Today 15:472-473

Kent ML, Andree KB, Bartholomew JL, El-Matbouli M and 12 others (2001) Recent advances in our knowledge of the myxozoa. J Eukaryot Microbiol 48:395-413

Lester RJG, Hallett SL, El-Matbouli M, Canning EU (1998) The case for naming actinosporeans using the Zoological Code. Parasitol Today 14:476-477

Lin D, Hanson LA, Pote LM (1999) Small subunit ribosomal RNA sequence of Henneguya exilis (Class Myxosporea) identifies the actinosporean stage from an oligochaete host. J Eukaryot Microbiol 46:66-68

Lom J, McGeorge J, Feist SW, Morris D, Adams A (1997) Guidelines for the uniform characterisation of the actinosporean stages of parasites of the phylum Myxozoa. Dis Aquat Org 30:1-9

Marques A (1984) Contribution á la connaissance des Actinomyxidies: ultrastructure, cycle biologique, systematique. These D'etat. Univ des Sciences et Techniques du Languedoc, Montpellier

McGeorge J, Sommerville C, Wootten R (1997) Studies of actinosporean myxozoan stages parasitic in oligochaetes from sediments of a hatchery where Atlantic salmon harbour Sphaerospora truttae infection. Dis Aquat Org 30:107-119

Molnár K, El-Mansy A, Székely C, Baska F (1999) Experimental identification of the actinosporean stage of Sphaerospora renicola Dyková et Lom, 1982 (Myxosporea: Sphaerosporidae) in oligochaete alternate hosts. J Fish Dis 22:143-153

Negredo C, Mulcachy MF (2001) Actinosporean infections in oligochaetes in a river system in southwest Ireland with descriptions of three new forms. Dis Aquat Org 46:67-77

Ormières R (1968) A propos de deux parasites d'oligochaetes de Besse: Diaspora (Coccidiomorpha Doflein, 1801) et Aurantiactinomyxon (Actinomyxidia Stolc, 1899). Ann St Biol Besse Chandesse 3:185-191

Ormières R, Frézil JL (1969) Aurantiactinomyxon eiseniellae n. sp., Actinomyxidie Parasite d'Eiseniella tetraedra Sav., (Oligochaeta - Lumbricidae). Protistologica 5:137-144

Oumouna M, Hallett SL, Hoffmann RW, El-Matbouli M (2003) Seasonal occurrence of actinosporeans (Myxozoa) and oligochaetes (Annelida) at a trout hatchery in Bavaria, Germany. Parasitol Res 89:170-184

Özer A, Wootten R, Shinn AP (2002) Survey of actinosporean types (Myxozoa) belonging to seven collective groups found in a freshwater salmon farm in northern Scotland. Folia Parasitol 49:189-210

Pote LM, Waterstrat P (1993) Motile stage of Aurantiactinomyxon sp. (Actinosporea: Triactinomyxidae) isolated from Dero digitata found in channel catfish ponds during outbreaks of proliferative gill disease. J Aquat Anim Health $5: 213-218$

Reed CC, Basson L, Van As LL (2002) Myxobolus species (Myxozoa), parasites of fishes in the Okawango River and Delta, Botswana, including descriptions of two new species. Folia Parasitol 49:81-88

Štolc A (1899) Actinomyxidies, nouveau groupe de Mesozoaires parent des Myxosporidies. Bull Int Acad Sci Boheme 22:1-12 
Styer EL, Harrison LR, Burtle GJ (1991) Experimental production of proliferative gill disease in channel catfish exposed to myxozoan-infected oligochaete, Dero digitata. J Aquat Anim Hlth 3:288-291

Székely C, El-Mansy A, Molnár K, Baska F (1998) Development of Thelohanellus hovorkai Achmerov, 1960 and Thelohanellus nikolskii Achmerov, 1955 (Myxosporea: Myxozoa) in oligochaete alternate hosts. Fish Pathol 33: $107-114$.

Székely C, Sitja-Bobadilla A, Alvarez-Pellitero P (2000) First report on the occurrence of an actinosporean stage (Myxozoa) in oligochaetes from Spanish freshwaters. Acta Vet Hung 48:433-441

Székely C, Yokoyama H, Urawa S, Timm T, Ogawa K (2003) Description of two new actinosporean types from a brook of Fuji Mountain, Honshu, and from Chitose River, Hokkaido, Japan. Dis Aquat Org 53:127-132

Timm T (1997) Freshwater Oligochaeta of some urban watercourses in the Russian Far East. Int Rev Ges Hydrobiol 82:437-467

Troullier A, El-Matbouli M, Hoffmann W (1996) A new look at the life cycle of Hoferellus carassii in the goldfish (Carassius auratus) and its relation to 'kidney enlargment disease' (KED). Folia Parasitol 43:173-187

Editorial responsibility: Wolfgang Körting, Hannover, Germany
Wolf K, Markiw ME (1984) Biology contravenes taxonomy in the Myxozoa: new discoveries show alternation of invertebrate and vertebrate hosts. Science 225:1449-1452

Xiao C, Desser SS (1998) Actinosporean stages of myxozoan parasites of oligochaetes from Lake Sasajewun, Algonquin Park, Ontario: new forms of echinactinomyxon, neoactinomyxum, aurantiactinomyxon, guyenotia, synactinomyxon and antonactinomyxon. J Parasitol 84: 1010-1019

Yokoyama H (1997) Transmission of Thelohanellus hovorkai Acmnerov, 1960 (Myxosporea: Myxozoa) to common carp, Cyprinus carpio, through the alternate oligochaete host. Syst Parasitol 36:79-84

Yokoyama H, Ogawa K, Wakabayashi H (1991) A new collection method of actinosporeans-a probable infective stage of myxosporeans to fishes-from tubificids and experimental infection of goldfish with the actinosporean, Raabeia sp. Fish Pathol 26:133-138

Yokoyama H, Ogawa K, Wakabayashi H (1993) Involvement of Branchiura sowerbyi (Oligochaeta: Annelida) in the transmission of Hoferellus carassii (Myxosporea: Myxozoa), the causative agent of kidney enlargement disease (KED) of goldfish Carassius auratus. Fish Pathol 28: 135-139

Submitted: January 20, 2004; Accepted: May 10, 2004 Proofs received from author(s): September 29, 2004 